\title{
Estimation of Cross Sections for Molecule-Cluster Interactions by using Artificial Neural Networks
}

\author{
Mustafa Böyükata $^{a}$, Yücel Koçyig̃it ${ }^{b}$, and Ziya B. Güvenç ${ }^{c}$ \\ ${ }^{a}$ Department of Physics, Erciyes University, 66100 Yozgat, Turkey \\ ${ }^{b}$ Department of Electric and Electronic Engineering, Celal Bayar University, 45140 Manisa, Turkey \\ ${ }^{c}$ Department of Electronic and Communication Engineering, Çankaya University, 06530 Ankara, Turkey
}

Received on 7 September, 2005

\begin{abstract}
The cross sections of $D_{2}(v, j)+N i_{n}(T), n=19$ and 20, collision systems have been estimated by using Artificial Neural Networks (ANNs). For training, previously determined cross section values via molecular dynamics simulation have been used. The performance of the ANNs for predicting any quantities in moleculecluster interaction has been investigated. Effects of the temperature of the clusters and the rovibrational states of the molecule are analyzed. The results are in good agreement with previous studies.
\end{abstract}

Keywords: Artificial Neural Networks; Molecular Dynamics; Clusters; Reactivity

\section{INTRODUCTION}

There is a strong motivation to investigate the size dependent chemical and physical properties of atomic clusters because of the development of electronic devices. Especially, hydrogen-metal systems have been studied extensively owing to their technological importance [1]. Cluster systems which are bridging between atoms on the one hand and bulks on the other offer an important opportunity to address a variety of structural and dynamical problems [2]. As the number of atoms grows, the theoretical and experimental difficulties grow much faster. Not surprisingly this leads to a number of many open questions. Even the middle size clusters have not been conclusively understood. In reactivity of clusters with molecules, e.g., the modes such as: temperature of the cluster, size, stability, symmetry, rovibrational states of the molecule, impact energies, impact sites, etc., play important roles and also some of these factors are strongly coupled. Therefore, the cluster-molecule reactivity problems are difficult and open questions [3-8]. Understanding of the dynamic and energetic factors of these reactions is important for elucidation of the microscopic mechanisms. As a result, the processes involved in chemisorptions of molecules on surface of small clusters are a research area of great interest for many years [3-24].

Various experimental [9-13] and theoretical [3-8,14-24] studies on the chemisorptions of hydrogen and deuterium on various metal clusters show that there can be large changes in the cluster reactivity within a very narrow size range. These investigations have provided valuable information about the reactivity of clusters. For example, Jellinek and Güvenç have pointed out maxima in the reactive cross sections (CSs), at about room temperature collision energy, of $D_{2}+N i_{13}$ system resulting from indirect dissociation mechanism. Similar trends were observed for different sizes of the clusters $[19,20]$ and on surface reactivity [25-28]. Additionally, in our resent work [29] a detailed quasiclassical trajectory study of the reactions of the $D_{2}(v, j)$ molecule with the larger clusters, $N i_{19}$ and $N i_{20}$, was reported at a wide range of temperatures of the clusters and of the rovibrational states of the molecule in order to reduce the scarcity in the literature. However, a de- tailed searching the $\mathrm{CS}$ calculations for all rovibrational states of the molecule and all interested temperatures of the clusters have not been realized due to the long run time consumption. Therefore, in the present work Artificial Neural Network (ANN) estimation has been performed to determine the CSs of the $D_{2}(v, j)+N i_{n}(T), n=19$ and 20, collision systems. Previously calculated CS values in Ref. [29] via molecular dynamics (MD) simulation have been used for training the ANNs.

The deterministic nature of a nonlinear system allows extracting its functional structure from a time series using appropriate nonlinear techniques [30]. By analogy with the human brain, neural network (NN) is massively parallel system that relies on the simple processors and dense arrangements of the individual interconnections of processing units in which information is passed [31]. These networks have demonstrated their ability to deliver simple and powerful solutions in areas that have challenged conventional computing. In the recent decade, ANNs have been widely and successfully used in many fields, including hysteresis modelling. This area has been developed to solve demanding pattern processing problems which were intractable or extremely cumbersome when implemented using traditional approaches [32].

In this paper the performance of ANNs for predicting the $\mathrm{CS}$ quantities in molecule-cluster interaction has been investigated. Furthermore, the effects of the rovibrational states of the molecule and the cluster temperature are also analyzed. The CSs are determined at $T=0,300,600,1200,1500,1800$ and $2100 \mathrm{~K}$ for both $N i_{19}$ and $N i_{20}$ clusters with the various vibrational $(v=0,1,2)$ and rotational $(j=0-20)$ states of the molecule, $D_{2}$. The ANN results are in good agreement with previous MD studies. Initial vibrational excitation of the molecule, for example, increases the reaction CSs more efficiently than the initial rotational excitation. In addition, the strong dependence of the reactive CSs on the collision energies, below $0.1 \mathrm{eV}$, is also observed with ANN estimation results. In the next section, outlines of the theoretical background and of ANN procedure are given. The numerical results and their discussions are presented in Sec. 3, and we conclude with a brief summary. 


\section{COMPUTATIONAL BACKGROUND AND ARTIFICIAL NEURAL NETWORKS}

The computational details for the MD results (to be used here to train ANN) are previously described in Ref. [29]. However, repeating the most relevant parts here may be necessary for the readers and clearness of the presentation. In that work the $D_{2}$ bombardment of the clusters at various impact parameters has been performed using a constant energy MD computer simulation. Hamilton's equations of motion were solved using Hamming's modified $4^{\text {th }}$ order predictor-corrector propagator with a step size of $5 \times 10^{-16}$ $s$ for the deuterium atoms in the system. The potential energy surface (PES) used in that simulation was formed by a four-body LEPS (London-Eyring-Polanyi-Sato) function accounts for $D-D$ and $D-N i$ interactions (for details see Refs. $[18,22])$. An embedded atom (EA) potential was used to describe the interaction between the $\mathrm{Ni}$ atoms [33]. With any specified collision energy and impact parameter on the cluster, 500 trajectories, corresponding to different initial relative orientations of the molecule with respect to the cluster, were run for each set of initial conditions in order to determine the dissociative chemisorption (DC) probability of the molecule. Through these probabilities the CSs of the DC are obtained. The minimum energy structures of the $N i_{19}$ and $N i_{20}$ clusters are a double-icosahedron formed by three pentagonal rings and a double-icosahedron with an additional atom on the surface, respectively. Detail information was presented in Ref. [29].

The ANN is an important information processing paradigm that was inspired by the way of biological nervous systems works, such as: the functionality of the brain. The key element of this paradigm is the novel structure of the information processing system. It is composed of a large number of highly interconnected processing elements (neurons) working in unison to solve specific problems. An ANN is configured for a specific application, such as pattern recognition or data classification, through a learning process like people. In biological systems learning involves adjustments of the synaptic connections that exist between the neurons. This is true for ANN's training process as well. A NN is represented by weighted interconnections between processing elements (PEs). These synaptic weights are the parameters that actually define the non-linear function performed by the NN. The process of determining such parameters is called training or learning [34], relying on the presentation of many training patterns. The ability to find correlation among apparently disconnected data and the tolerance to noisy data are the main features of the ANNs. For a real problem, any ANN must be trained at the beginning. Here, we have employed Back-Propagation (BP) training algorithm $[32,35]$. Thus, NNs are inherently adaptive, conforming to the imprecise, ambiguous and faulty nature of real-world data.

The BP algorithm is the most widely used NN because of its relatively simplicity and universal approximation capacity [36]. The BP algorithm defines a systematic way to update the synaptic weights of multi-layer perceptron (MLP) networks. The supervised learning is based on the gradient de-

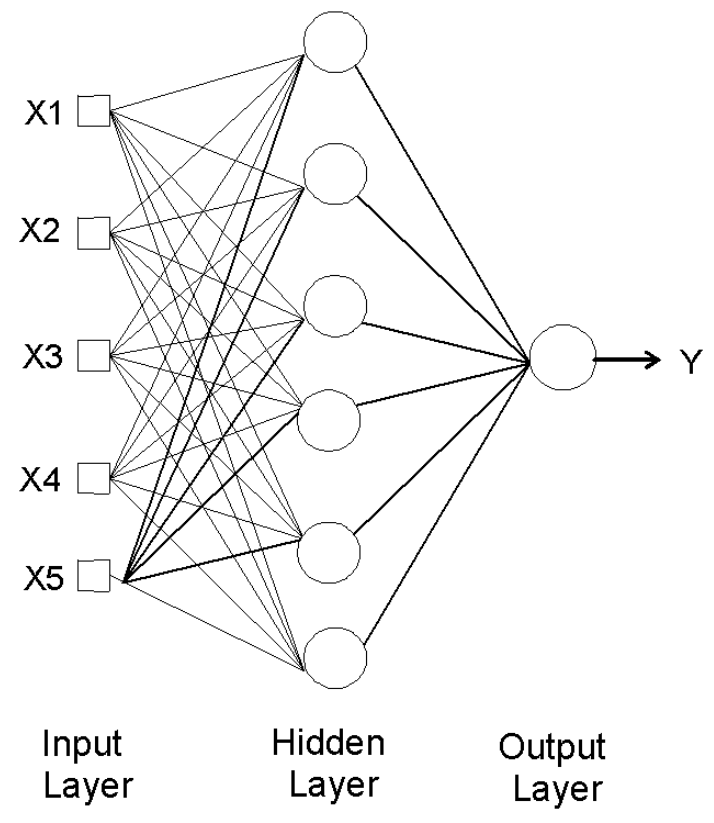

FIG. 1: General structure of MLP neural network architecture.

scent method, minimizing the global error on the output layer. The learning algorithm is performed in two stages [37]: feedforward and feed- backward. In the first phase, the inputs are propagated through the layers of processing elements, generating an output pattern in response to the input pattern presented. In the second phase, the errors calculated in the output layer are then back propagated to the hidden layers where the synaptic weights are updated to reduce the error. This learning process is repeated until the output error value, for all patterns in the training set, are below a specified value. The definition of the network size (the number of hidden layers and of neurons in each layer) is a compromise between generalization and convergence. Convergence is the capacity of the network to learn the patterns on the training set, and generalization is the capacity to respond correctly to new patterns. The idea is to implement the smallest network possible, so it is able to learn all patterns, and at the same time, provide good generalization. However, a very long training process, with problems such as local minima; and the restriction of learning only static input -output mappings are two limitations of BP [37]. In this study a three-layered ANN is used and trained with the BP algorithm [38]. Detailed information about ANNs procedure has been given in Refs. [37-39] and related references therein.

The BP network used is composed of an input layer, a hidden layer and an output layer (Fig. 1). The number of neurons in the hidden layer has been determined via experimentation. The experimental results show that the optimum number of hidden neurons was six. In the present work the number of class is equal to 1 , which is the number of neuron in the output layer. As mentioned above, to overcome BP limitations, like local minima, an adaptative learning rate (0.3) and a momentum constant $(0.7)$ has been used. The stopping criteria for the network training are the sum of squared error $(0.02)$ 


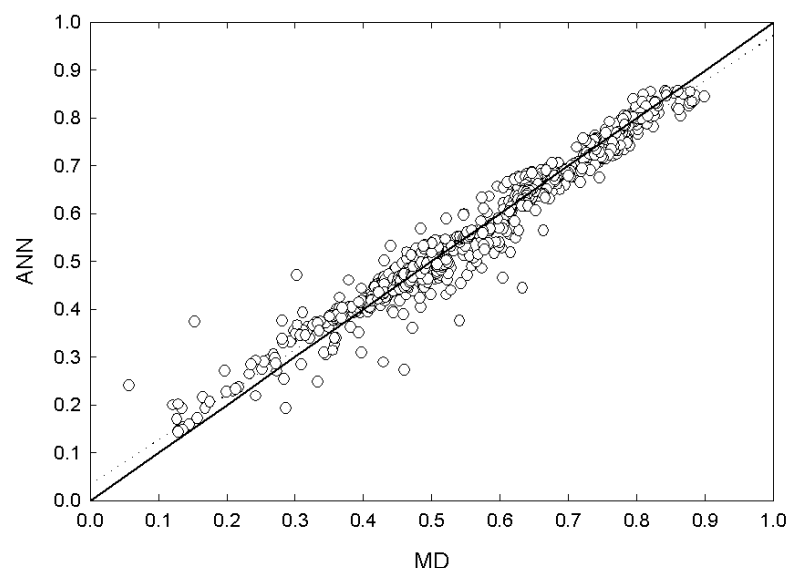

FIG. 2: Estimated ANN results versus used MD data [29] for training.

and a maximum number of epochs (10000).

\section{RESULTS AND DISCUSSIONS}

In order to see the agreements of the ANN estimated $\mathrm{CS}$ values with the used MD data they are compared in Fig. 2 , which are normalized between 0 and 1 . As it has presented in the figure, the ANNs can learn perfectly the relationships between the input variables (X1; cluster size, $\mathrm{X} 2$; cluster temperature, $\mathrm{X} 3$ and $\mathrm{X} 4$; vibrational and rotational states, respectively, and X5; collision energies), and the output (Y; CSs). The number of MD data [29] and their ranges that are used as input for the training of the ANN are presented in Table I. For one of the two X1 values $\left(N i_{19}\right) 364$ data have been used which were calculated for five different temperatures (X2) of the cluster with the ground state of the molecule $(v=0, j=0),(\mathrm{X} 3, \mathrm{X} 4)$, with various collision energies (X5) up to $1.0 \mathrm{eV}$ and for $2202 \mathrm{~K}$ at $v=0$ rotational states (X4) $j=0-11,13,15$ and at $v=1, j=0$. For the $\mathrm{X} 1=20$ (i.e., $\left.N i_{20}\right), 644$ samples consisting of $(v=0, j=0,3)$ and $(v=1, j=0)$ states of the molecule at $0 \mathrm{~K}$ cluster and $(v=0, j=0,1,3,5,7,9,10,12,15,20),(v=1, j=0,1,3,5,9)$ and $(v=2, j=0,1,3,5,7)$ at $291 K$ have been used. Totally, 1008 data have been evaluated to improve the ANNs and tested with the used MD data. Then it is understood that the ANNs are able to produce reliable values with $0.03 \%$ mean error in this particular analysis.

The contour graphs in Fig. 3 present the reactive CSs of the $D_{2}(v, j)$ molecule with the $N i_{19}(T=300 \mathrm{~K})$ cluster as a function of the collision energy (up to $1.0 \mathrm{eV}$ ) and rotational states $(j=0-20)$, for three vibrational modes; $v=0, v=1$ and $v=2$ (respectively from top to bottom). In the low energy region (starting from near zero) reactivity increases rapidly and reaches to a maximum value about $0.05 \mathrm{eV}$ for all vibrational cases. As the collision energy increases further, the CSs decrease and have minimum values. Finally, for the higher collision energies the curves increase slowly. The maximum

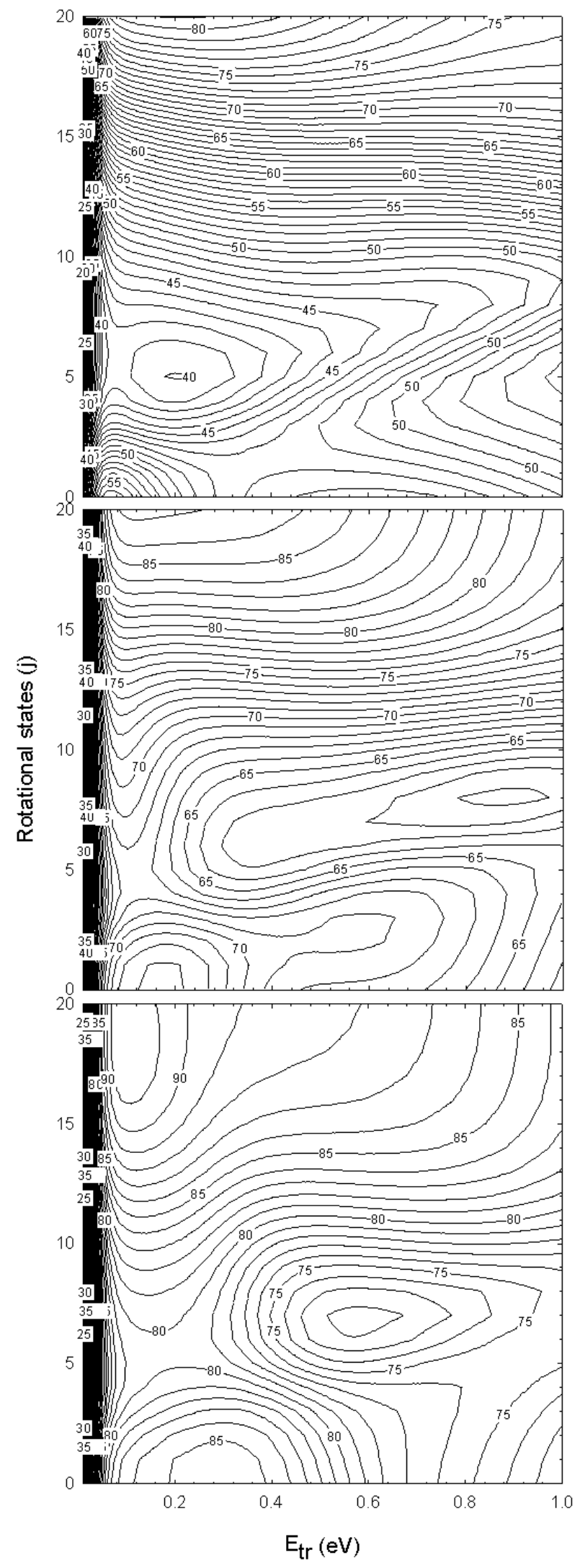

FIG. 3: Cross sections for $D_{2}(v, j)+N i_{19}(300 K)$ as functions of rotational states and collision energy for three vibrational modes; $v=$ 0 (top), $v=1$ (middle) and $v=2$ (bottom), respectively. 
TABLE I: The MD data ranges [29] that used as input to train the ANNs.

\begin{tabular}{cccccc}
\hline \hline $\mathrm{X} 1$ & $\mathrm{X} 2$ & $\mathrm{X} 3$ & $\mathrm{X} 4$ & $\mathrm{X} 5$ & Number of data \\
\hline 19 & 164 & 0 & 0 & $0.01-1.0$ & 28 \\
19 & 775 & 0 & 0 & $0.01-1.0$ & 28 \\
19 & 1351 & 0 & 0 & $0.01-1.0$ & 28 \\
19 & 1684 & 0 & 0 & $0.01-1.0$ & 28 \\
19 & 2202 & 0 & $0-11,13,15$ & $0.01-1.0$ & 224 \\
19 & 2202 & 1 & 0 & $0.01-1.0$ & 28 \\
20 & 0 & 0 & 0,3 & $0.01-1.0$ & 56 \\
20 & 0 & 1 & 0 & $0.01-1.0$ & 28 \\
20 & 291 & 0 & $0,1,3,5,7,9,10,12,15,20$ & $0.01-1.0$ & 280 \\
20 & 291 & 1 & $0,1,3,5,9$ & $0.01-1.0$ & 140 \\
20 & 291 & 2 & $0,1,3,5,7$ & $0.01-1.0$ & 140 \\
\hline \hline
\end{tabular}

peaks in the low energy region represent a formation of the molecule-cluster complex, that is, the molecule is attached to the surface of the cluster and can orient itself to search for the "active-site" to break its bond. This "resonance" enhances the reactivity as seen from Fig. 3. As energies increase the molecule has less and less time on the surface to orient itself (the life-time of the molecule-cluster complex decreases), therefore, it cannot follow the minimum energy path to lower the effect of the repulsive part of the PES. Hence, in parallel, the reactive CSs drop as collision energies increase to the higher values. Towards $1.0 \mathrm{eV}$ all the CS slightly increase and merge gradually to a similar value. For high temperatures of the clusters (the liquid-like forms) on the other hand, this increment is somewhat larger. They also merge gradually to a similar value. This means that if the collision temperature is lower than or similar to the room temperature, then the temperature $(T)$ of the cluster plays strong roll in the reactivity (for details see Refs. 4-8).

The cluster is at room temperature, $T=300 \mathrm{~K}$. In the low collision energy region $\left(E_{t r}<0.1 \mathrm{eV}\right)$ the dynamics of the $\mathrm{DC}$ is also complicated due to the curve crossings. The MD results [29] showed that at the lowest energy considered, the $(v=1, j=0)$ state is the most reactive one, and the others are ordered in the decreasing order as $(v=0, j=0),(v=0, j=$ $3)$, and $(v=0, j=10)$, respectively. This shows that vibrationally excited molecule is more reactive than the rotationally excited molecules. On the other hand, at the collision energy of $E_{t r}=0.03 \mathrm{eV}$, the $(v=0, j=0)$ state is the most reactive one, and the others are ordered in the decreasing order as $(v=1, j=0),(v=0, j=3)$, and $(v=0, j=10)$, respectively. Above this energy, the $(v=1, j=0)$ is always the most reactive state, and the $(v=0, j=10)$ is the least reactive state up to $E_{t r}=0.1 \mathrm{eV}$ despite the fact that the internal energy of the $(v=0, j=10)$ state is higher than that of the $(v=1, j=0)$ state. This shows that energy should be put into the vibrational modes not into the rotational states to increase the reactivity. As a result of that study, Ref. [29], below $0.1 \mathrm{eV}$ energy rotational excitation hinders the reactivity, i.e., the higher the rotational state, lower the cross section. At the higher collision energies (above $0.3 \mathrm{eV}$ ) higher rotational excitation yields higher reactivity. The "resonance" phenomenon is observed clearly for the $(v=0, j=0)$ state, and it is less pronounced for the other excited states. Similarly, in this work ANN determined data produces all interested specific regions. There are two typical saddle points near $(0.08$ $e V, j=6)$ and $(0.40 e V, j=2)$ values for $v=0$. Moreover, for $E_{t r}=0.20 \mathrm{eV}$ and $j=5$ values a minimum CS region has been observed. For $v=1$ and $v=2$, the similar minima are observed $(0.88 \mathrm{eV}, j=8)$ and $(0.58 \mathrm{eV}, j=7)$, respectively. However, their values are not very lover because the excitations in the vibrational mode of the molecule increase the reactivity.

Now we discuss the size and the temperature effect on the $\mathrm{CS}$ values by analyzing the minimum values of CSs. As the size of the cluster increases by a small amount, there is not a big difference between 19 and 20 atom clusters as shown in Table II. Their structural geometries are also closer each other and this leads similar trends in CSs which are in good coincidence with MD investigations [29]. As shown in the table increase in the temperature of the clusters causes high reactivity and the values of the CSs increase. Interestingly, for all cases this minima are observed at $j=5$ for $v=0$. However, for $v=1$ at $j=9$ the minima is observed for the high temperatures, greater than $1500 K$, despite of $j=8$ for other temperatures. For $v=2$ this typical rotational state is $j=8$ for all temperature except $0 \mathrm{~K}$.

\section{CONCLUDING REMARK}

The main goal of this study is to test the performance of ANNs for predicting any quantities in any molecule cluster interaction studies. It has been observed that it can be used as an efficient tool to estimate the CS values for $D_{2}+N i_{19}$ and $\mathrm{D}_{2}+\mathrm{Ni}_{20}$ collisions.

The DC-CS determined (by ANNs) from the rovibrationally excited molecules with the solid- and liquid-like forms of the clusters strongly depend on to the collision energy, especially below $0.2 \mathrm{eV}$. At the higher energies this dependence is weaker. In this study the dynamics of the DCs with respect to the $v, j$, and $T$ are also complicated in the low collision energy region (below $0.1 \mathrm{eV}$ ). In general, the rotational excitations and the high temperature of the cluster hinder the reactivity. This is more pronounced especially below 
TABLE II: Determined rotational states and collision energy regions in which the minimum CSs are observed at different temperature of the clusters.

\begin{tabular}{cccccccc}
\hline \hline \multirow{2}{*}{$T(K)$} & $v$ & $j$ & $E_{t r}$ & $\mathrm{CSs}$ & & \multicolumn{3}{c}{$N=20$} \\
\hline 0 & 0 & 5 & 0.22 & 40.33 & 5 & 0.22 & 40.33 \\
& 1 & 8 & 0.88 & 61.06 & 8 & 0.88 & 61.20 \\
& 2 & 0 & 1.00 & 72.00 & 0 & 1.00 & 72.13 \\
300 & 0 & 5 & 0.20 & 39.93 & 5 & 0.20 & 40.11 \\
& 1 & 8 & 0.88 & 60.66 & 8 & 0.88 & 60.81 \\
& 2 & 7 & 0.58 & 71.62 & 7 & 0.58 & 71.66 \\
600 & 0 & 5 & 0.18 & 39.16 & 5 & 0.19 & 39.71 \\
& 1 & 8 & 0.89 & 59.92 & 8 & 0.89 & 60.42 \\
& 2 & 7 & 0.56 & 70.67 & 7 & 0.57 & 71.17 \\
900 & 0 & 5 & 0.18 & 39.16 & 5 & 0.18 & 39.33 \\
& 1 & 8 & 0.89 & 59.92 & 8 & 0.90 & 60.07 \\
& 2 & 7 & 0.56 & 70.67 & 7 & 0.56 & 70.71 \\
\hline
\end{tabular}

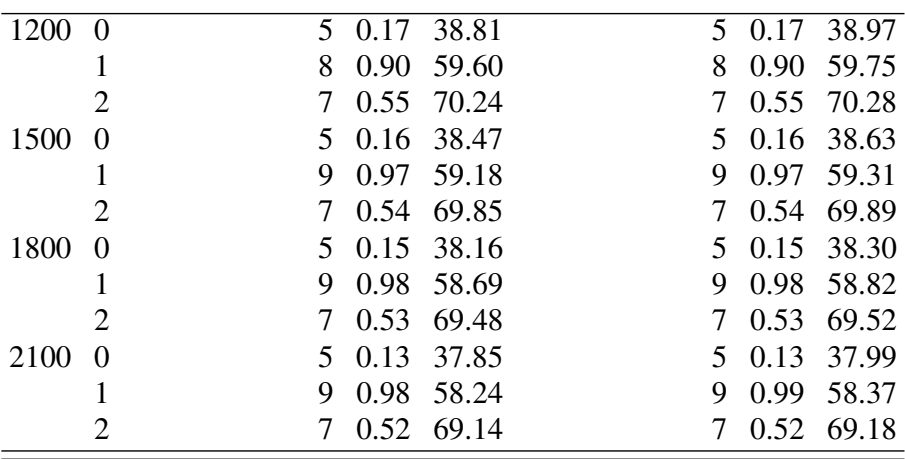

$0.1 \mathrm{eV}$.

Notice that no extra effort is necessary to compute the CSs for all the rovibrational states and the initial collision energies. After getting a sufficient set of data for the training process, ANNs can be used to predict for all the interested initial conditions of the system. In conclusion, we believe that ANNs introduces new ideas that may help researchers to tackle optimization problems that, so far, have not been conveniently investigated.

\section{Acknowledgements}

This work was supported by Erciyes University Research Fund (Project Number: FBA.03.29).
[1] B. Chen, M. A. Gomez, and J. D. Doll, J. Chem. Phys. 108, 4031 (1998)

[2] B. Chen, M. A. Gomez, M. Sehl, J. D. Doll, and D. L. Freeman, J. Chem. Phys. 105, 9686 (1996).

[3] J. Jellinek and Z.B. Güvenç, Z. Phys. D 19, 371 (1991).

[4] J. Jellinek and Z. B. Güvenç, in Mode Selective Chemistry (eds. J. Jortner et al.) (Netherlands, Kluwer Academic Publishers 1991) p. 153.

[5] J. Jellinek and Z. B. Güvenç, in Physics and Chemistry of Finite Systems: From Clusters to Crystals (eds. P. Jena et al.) (Netherlands, Kluwer Academic Publishers 1992) Vol. II p. 1047.

[6] J. Jellinek and Z. B. Güvenç, Z. Phys. D 26, 110 (1993).

[7] J. Jellinek and Z. B. Güvenç, in Topics in Atomic and Nuclear Collisions (eds. B. Remaund et al.) (New York, Plenum Press 1994) p. 243

[8] J. Jellinek and Z.B. Güvenç, in The Senergy Between Dynam- ics and Reactivity at Clusters and Surfaces, ed. L. J. Farrugia (Kluwer Academic Publishers, Dordrecht, 1995) p. 217.

[9] W.F. Hoffman III, E.K. Parks, G.C. Nieman, L.G. Pobo, and S.J. Riley, Z. Phys. D 7, 83 (1987).

[10] L. Zhu, J. Ho, E.K. Parks, and S.J. Riley, J. Chem. Phys. 98 2798 (1993).

[11] H.J. Robota, W. Vielhaber, M.C. Lin, J. Segner, and G. Ertl, Surf. Sci. 155, 101 (1985).

[12] A.V. Hamza, R.J. Madix, J. Chem. Phys. 89, 5381 (1985)

[13] D.O. Hayward, A.O. Taylor, Chem. Phys. Lett. 124, 264 (1986).

[14] M.D. Morse, M.E. Geusic, J.R. Heath, and R.E. Smalley, J. Chem. Phys. 83, 2293 (1985).

[15] S.C. Richsmeier, E.K. Parks, K. Liu, L.G. Pobo, and S.J. Riley, J. Chem. Phys. 82, 3659 (1985).

[16] P.E.M. Seigbaum, M.R.A. Bloomburg, and C.W. Baucschlicher, Jr, J. Chem. Phys. 81, 2103 (1984). 
[17] C.Y. Lee, A.E. De Pristo, J. Chem. Phys. 84, 485 (1986).

[18] K. Raghavan, M.S. Stave, and A.E. DePristo, J. Chem. Phys. 91, 1904 (1989).

[19] P. Durmuş, M. Böyükata, S. Özçelik, Z. B. Güvenç, and J. Jellinek, Surf. Sci. 454, 310 (2000).

[20] M. Böyükata, Z. B. Güvenç, S. Özçelik, P. Durmuş, and J. Jellinek, Int. J. Quantum Chem. 84, 208 (2001).

[21] J. Jellinek and I.L. Garzon, Z. Phys. D 20, 239 (1991).

[22] M.S. Stave and A.E. DePristo, J. Chem. Phys. 97, 3386 (1992).

[23] R. Fournier, M.S. Stave, and A.E. DePristo, J. Chem. Phys. 96, 1530 (1992).

[24] Y.L. Alvarez, G.E. Lopez, and A.J. Cruz, J. Chem. Phys. 107, 1420 (1997).

[25] C.T. Rettner and D.J. Auerbach, Chem. Phys. Lett. 253, 236 (1996).

[26] A. Gross, Surf. Sci. Rep. 32, 291 (1998).

[27] K.D. Rendulic, G. Anger, A. Winkler, Surf. Sci. 208, 404 (1989).

[28] M. Böyükata, Z. B. Güvenç, B. Jackson, and J. Jellinek, Int. J. Quantum Chem. 84, 48 (2001).

[29] M. Böyükata, Z. B. Güvenç, S. Özçelik, P. Durmuş, and J. Jellinek, Int. J. Mod. Phys. C 16, 295 (2005).

[30] S. Ortín, J.M. Gutiérrez, L. Pesquera, and H. Vasquez, Physica
A 351, 133 (2005).

[31] P. Treleaven, M. Pacheco, and M. Vellasco, IEEE Micro. 9, 8 (1989).

[32] R. Hecht-Nielsen, Neurocomputing (Addison-Wesley, San Diego, 1989).

[33] A.F. Voter and S.P. Chen, Mater. Res. Soc. Symp. 82, 175 (1987).

[34] C.M. Bishop, Rev. Sci. Intrum. 65, 1803 (1994).

[35] J. Hertz, A. Krogh, and R.G. Palmer, Introduction to the Theory of Neural Computation, Addison-Wesley, Reading, MA (1991).

[36] K. Hornik, M. Stinchcombe, and H. White, Neural Networks 2, 359 (1989).

[37] S. Haykin, Neural Networks: A Comprehensive Foundation, Prentice Hall, Upper Saddle River, NJ, (1999).

[38] B. Karlik, M.O. Tokhi, and M. Alci, IEEE Trans. Biomed. Eng. 50, 1255 (2003).

[39] R.Q. Feitosa, M.M.B.R. Vellasco, D.T. Oliveira, D.V. Andrade, and S.A.R.S. Maffra, in Proceedings of the $4^{\text {th }}$ World Multiconference on Systemics, Cybernetics and Informatics (SCI'2000) and the 6th International Conference on Information Systems Analysis and Synthesis (ISAS'2000), Orlando, USA, (2000) p. 73. 